\title{
A large HST program: effective temperatures of cataclysmic variable white dwarfs
}

A. F. Pala*1, B. T. Gänsicke ${ }^{1}$, K. Beuermann ${ }^{2}$, L. Bildsten ${ }^{3}$, D. De Martino ${ }^{4}$, P. Godon ${ }^{5}$, A. A. Henden ${ }^{6}$, I. Hubeny ${ }^{7}$, C. Knigge ${ }^{8}$, K. S. Long ${ }^{9}$, T. R. Marsh ${ }^{1}$, J. Patterson ${ }^{10}$, M. R. Schreiber ${ }^{11}$, E. M. Sion ${ }^{5}$, P. Szkody ${ }^{12}$, D. Townsley ${ }^{13}$, M. Zorotovic ${ }^{11}$

1Department of Physics, University of Warwick, Coventry, CV4 7AL, UK

${ }^{2}$ Institut für Astrophysik, Georg-August-Universität, D-37077 Göttingen, Germany

${ }^{3}$ Department of Physics, Broida Hall, University of California, Santa Barbara, CA 93106, USA

${ }^{4} I N A F$ áĹŠ Osservatorio Astronomico di Capodimonte, Napoli, I-80131, Italy

${ }^{5}$ Astronomy \& Astrophysics, Villanova University, Villanova, PA 19085, USA

${ }^{6}$ American Association of Variable Star Observers, Cambridge, MA 02138, USA

${ }^{7}$ Steward Observatory, The University of Arizona, Tucson, AZ 85721, USA

${ }^{8}$ School of Physics and Astronomy, University of Southampton, Southampton, SO17 1BJ, UK

${ }^{9}$ Space Telescope Science Institute, 3700 San Martin Drive, Baltimore, MD 21218, USA

${ }^{10}$ Department of Astronomy, Columbia University, New York, NY 10027, USA

${ }^{11}$ Instituto de Física y Astronomía, Universidad de Valparaíso, 2360102 Valparaiso, Chile

${ }^{12}$ Department of Astronomy, University of Washington, Seattle, WA 98195-1580, USA

${ }^{13}$ Department of Physics and Astronomy, The University of Alabama, Tuscaloosa, AL 35487, USA

E-mail: A.F.Pala@warwick.ac.uk

We present $H S T / \mathrm{COS}$ spectroscopy for 40 cataclysmic variables $(\mathrm{CVs})$, observed as part of a 122 orbit $H S T$ program. In $31 \mathrm{CVs}$, the white dwarf (WD) is recognisable through its broad Ly $\alpha$ absorption profile and we measure the WD effective temperatures ( $T_{\text {eff }}$ ) by fitting the HST data of these 31 systems assuming $\log g=8.35$, which corresponds to the average mass for $\mathrm{CV}$ WDs $\left(0.8 \mathrm{M}_{\odot}\right)$. Our results nearly double the number of $\mathrm{CV}$ WDs with an accurate temperature measurement.

We find that the $T_{\text {eff }}$ of CV WDs above the period gap are, on average, higher and exhibit much more scatter compared to those below the gap. This behaviour broadly agrees well with theoretical predictions, reflecting different angular momentum loss mechanisms driving $\mathrm{CV}$ evolution above and below the period gap. Although our results show the expected decrease in $T_{\text {eff }}$ below the period gap, they are not clustered in the predicted narrow track but present a relatively large spread near the period minimum. These findings are in contrast with the results from population synthesis and may point to some shortcomings in the CV evolutionary models. Reaching the minimum period, $\mathrm{CVs}$ are expected to evolve back towards longer periods with mean accretion rates $\dot{M} \lesssim 10^{-11} \mathrm{M}_{\odot} \mathrm{yr}^{-1}$, corresponding to $T_{\text {eff }} \lesssim 10000 \mathrm{~K}$. We do not identify these systems in our survey, suggesting that none of them are strong "period bouncer" candidates, and this major component of the predicted $\mathrm{CV}$ population still remains elusive to observations.

The Golden Age of Cataclysmic Variables and Related Objects - III, Golden2015

7-12 September 2015

Palermo, Italy 


\section{Introduction}

Cataclysmic variables (CVs) are close interacting binaries containing a white dwarf (WD) accreting mass from a Roche-lobe filling low-mass companion star (Warner, 1995), giving rise to an accretion disc around the $\mathrm{WD}^{1}$.

The evolution and the final fate of CV WDs are, as for all types of accreting WD, of key interest in the framework of Type Ia Supernovae (SNe Ia) progenitors. Although early work showed the average mass of CV WDs to lie in the range $\left\langle M_{\mathrm{WD}}\right\rangle \simeq 0.8-1.2 \mathrm{M}_{\odot}$ (Warner 1973, Ritter 1987), higher than that for isolated WDs $\left\langle M_{\mathrm{WD}}\right\rangle \simeq 0.6 \mathrm{M}_{\odot}$ (Liebert et al. 2005, Koester et al. 1979, Kepler et al. 2007), historically CVs have not been considered as SNe Ia progenitors. This is because most models for classical novae - i.e. the thermonuclear ignition of the accreted hydrogen - predict that not only most (if not all) of the accreted matter (Prialnik \& Kovetz, 1995) but also some of the underlying core (Epelstain et al., 2007) would be ejected during the eruption, preventing mass growth. Nevertheless, Zorotovic et al. (2011) showed that CV WD masses are genuinely higher than both those of their detached progenitors and those of single white dwarfs, suggesting that CV WDs may grow in mass. It is therefore fundamental to improve our understanding of the long-term evolution of CVs in order to investigate their potential role as $\mathrm{SNe}$ Ia progenitors.

$\mathrm{CV}$ evolution is driven by angular momentum loss (AML) which results in continuous mass loss from the donor star, and sets the mass accretion rate $\dot{M}$. It has been suggested (Rappaport et al. 1983, Paczynski \& Sienkiewicz 1983, Spruit \& Ritter 1983) that the evolution of CVs can be explained combining (at least) two different AML mechanisms: magnetic braking, which dominates for orbital periods $P_{\text {orb }} \gtrsim 3 \mathrm{hr}$ with typical mass transfer rates of $\dot{M} \sim 10^{-9}-10^{-8} \mathrm{M}_{\odot} \mathrm{yr}^{-1}$ (Spruit \& Ritter, 1983), and gravitational wave radiation, the dominant AML for $P_{\text {orb }} \lesssim 3 \mathrm{hr}$ with typical $\dot{M} \sim 5 \times 10^{-11} \mathrm{M}_{\odot} \mathrm{yr}^{-1}$ (Patterson, 1984). As angular momentum is removed from the orbit, the system shrinks and the orbital period decreases, therefore $\mathrm{P}_{\text {orb }}$ provides an indicator of the accretion rate and the evolutionary stage of the system. A more precise measurement of the mean accretion rate can be derived from the WD effective temperature $\left(T_{\text {eff }}\right)$ : its value is set by compressional heating of the accreted material, providing a constraint on the time-averaged mass-transfer rate $\langle\dot{M}\rangle$ (averaged over the thermal time-scale of the WD envelope, $10^{3}-10^{5} \mathrm{yr}$ ) and is one of the best available tests for the present models of CV evolution (Townsley \& Bildsten, 2003).

While there are now over 1100 CVs with an orbital period determination (Ritter \& Kolb 2003, 7th Edition, Release 7.21, March 2014), relatively little is know about their accreting white dwarfs: only 35 have an accurate mass determination (Zorotovic et al., 2011) and a reliable $T_{\text {eff }}$ measurement is available for only $43 \mathrm{CV}$ WDs (Townsley \& Gänsicke, 2009). To improve our knowledge of $\mathrm{CV}$ evolution, it is essential to increase the number of objects with an accurate $T_{\text {eff }}$ and mass measurement. Since CV WDs are relatively hot objects $\left(T_{\text {eff }} \gtrsim 10000 \mathrm{~K}\right)$ their spectral energy distribution peaks in the ultraviolet (UV). At these wavelengths, the contamination from the accretion flow and the secondary star are often negligible compared to their contribution in the optical, therefore space-based UV observations are necessary for a WD $T_{\text {eff }}$ determination (Szkody et al. 2002, Gänsicke et al. 2006, Sion et al. 2008, and many others). For this purpose, we carried out a

\footnotetext{
* Speaker.

${ }^{1}$ This is true only for non-magnetic systems and intermediate polars. Here, we only consider non-magnetic systems.
} 
large Hubble Space Telescope (HST) program in Cycle 20 (122 orbits), in which we obtained highresolution ultraviolet spectroscopy of $40 \mathrm{CV}$ WDs with the Cosmic Origin Spectrograph (COS).

The targets were selected to sample the entire orbital period range of the galactic CV population; three objects with previous $T_{\text {eff }}$ measurements were included for comparison with our results. In particular, we selected one eclipsing system to compare our spectroscopic analysis with results obtained from modelling the eclipse light curve (e.g. Littlefair et al. 2006, 2008), which is the most commonly used method for determining WD $T_{\text {eff }}$ from ground based observations.

Here we present the $H S T$ observations and the results of our spectral analysis, which almost doubles the number of objects with reliable $T_{\text {eff }}$ measurements. In the the next years, the ESA Gaia mission will provide accurate parallaxes for all the targets in our sample. From the knowledge of the temperature and the distance, the WD mass can then be estimated assuming a mass-radius relationship, doubling also the number of systems with an accurate mass determination. This enlarged sample of well-characterised CV WDs will be used to answer the question whether accreting CV WDs grow in mass.

\section{Observations}

The data were collected in 122 HST orbits from October 2012 to March 2014. Each CV was observed with COS for two to five consecutive spacecraft orbits. The data were obtained using the Primary Science Aperture (PSA) in TIME TAG mode, which also allows construction of light curves. We used the G140L grating at the central wavelength of $1105 \AA$ and the far-ultraviolet (FUV) channel, covering the wavelength range of $1105-2253 \AA$. The detector sensitivity quickly decreases in the red portion of the spectrum, reducing the actual useful range to $\simeq 1150-1730 \AA$, with a nominal resolution of $\mathrm{R} \sim 3000$. The data from all the orbits were summed to produce a single ultraviolet (UV) spectrum for each object. The COS FUV detector consists of a photoncounting micro-channel plate detector which is subject to damage by excessive illumination.

An important characteristic of many CVs is that periods of quiescence, in which the accretion onto the WD is greatly reduced, are interrupted by bright outbursts (e.g. as in Dwarf Novae).. These arise from a thermal instability in the disc causing a variation in the mass transfer rate through the disc (Osaki, 1974). During an outburst, CVs brighten typically by 2-5 magnitudes, occasionally up to 8 magnitudes (Warner, 1995). This increase in luminosity occurs rapidly, on the time-scale of about a day, first at optical wavelengths and only hours to a day later in the UV (King, 1997). Therefore CVs can easily reach, and exceed, the COS detector safety threshold. These events are unpredictable and to avoid damaging the $H S T$ detector an intensive monitoring of each target was required before the observations. This monitoring program was carried out by the global amateur community (AAVSO, CBA and many others), and only their outstanding support has made this HST survey possible.

After an outburst, the WD does not cool immediately to its quiescence temperature since it has been heated by the increased infall of material. The cooling time is related to the outburst amplitude and can vary from days to weeks, up to years (Gänsicke \& Beuermann 1996, Slevinsky et al. 1999, Piro et al. 2005). Therefore the effective temperature measured in a system in which an outburst has occurred recently provides only an upper limit for its quiescence effective temperature and, consequently, for $\langle\dot{M}\rangle$. 


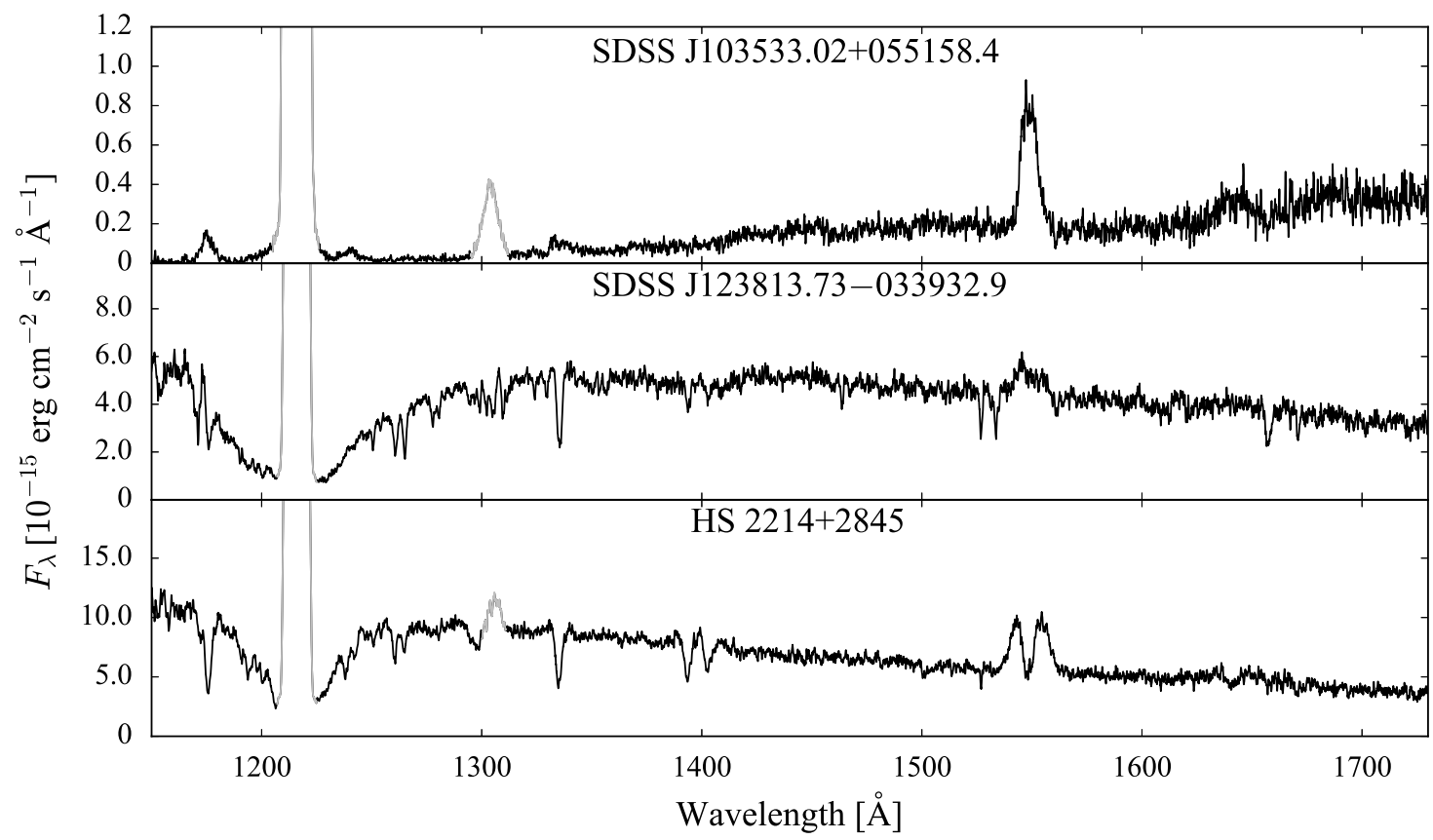

Figure 1: HST/COS spectra for three objects in which the WD dominates the far-ultraviolet. The temperatures are, top to bottom: $\simeq 11000 \mathrm{~K}, \simeq 18000 \mathrm{~K}, \simeq 26000 \mathrm{~K}$. The geocoronal emission lines of $\operatorname{Ly} \alpha(1216 \AA)$ and O I (1302 $\AA$ ) are plotted in grey.

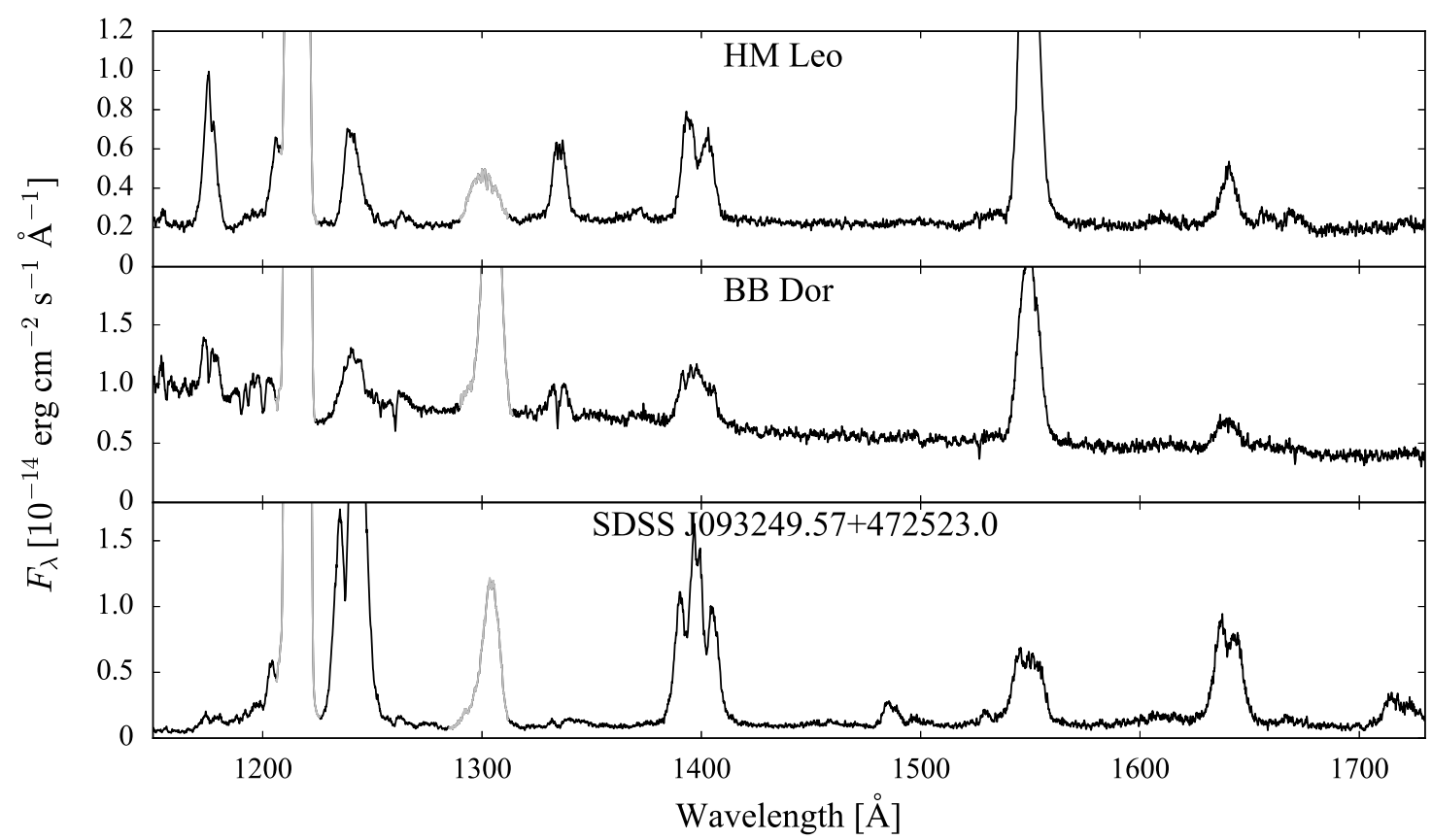

Figure 2: HST/COS spectra of three objects dominated by emission from the accretion flow, outshining the WD. The geocoronal emission lines of Ly $\alpha(1216 \AA)$ and O I (1302 $⿱$ ) are plotted in grey. 

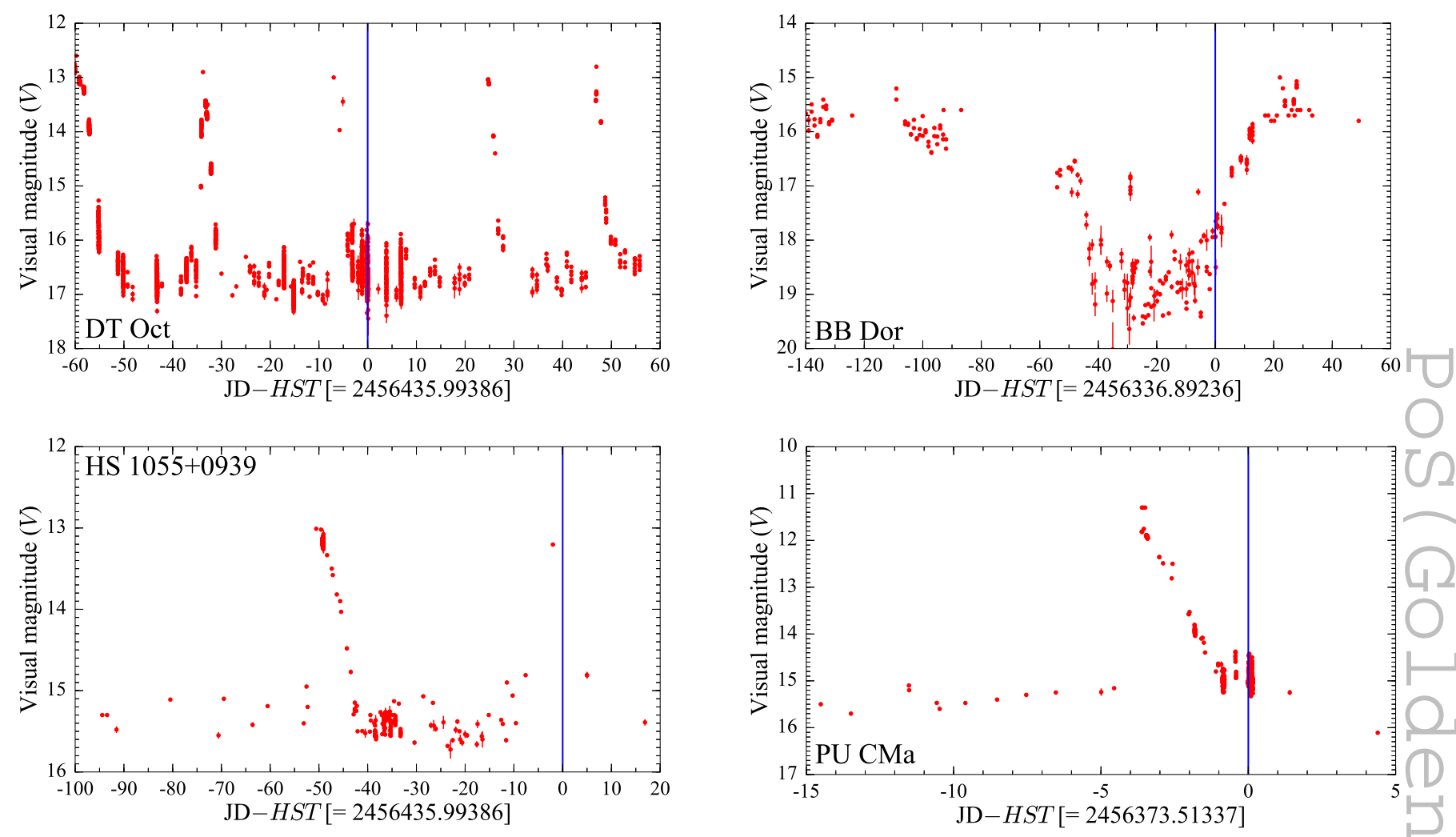

Figure 3: Light curves for four targets in our sample which have been observed close to an outburst or in a high state. The blue line represents the date of the HST observation. The data have been retrieved from the AAVSO database.

We conducted the survey on a sample of 40 objects. Figure 1 shows several sample UV spectra of quiescent CVs in which the WD dominates the emission, as seen from the broad Ly $\alpha$ absorption centered on $1216 \AA$. The quasi-molecular absorption bands of $\mathrm{H}_{2}^{+}$and of $\mathrm{H}_{2}$ are also visible at $\simeq 1400 \AA$ and $\simeq 1600 \AA$ for $T \lesssim 19000 \mathrm{~K}$ and $T \lesssim 13500 \mathrm{~K}$, respectively (Nelan \& Wegner 1985 , Koester et al. 1985). The shape of the Ly $\alpha$ line changes with $T_{\text {eff }}$, becoming more defined and narrower in the hotter WDs, while the continuum slope of the spectrum becomes steeper. We could not identify the WD signature in nine systems: seven of them experienced an outburst shortly before the HST observation (see Figure 3) while the remaining two systems are VY Scl stars. VY $\mathrm{Scl}$ stars are characterised by high mean accretion rates which keep the disc permanently in the hot equivalent to a dwarf nova in outburst. In this high state, the disc outshines the WD even in the far UV. However, occasionally the accretion rate drops (low state) and unveils the WD. The VY Scl stars in our sample (OR And and BB Dor) were caught in high state, preventing the WD identification. The spectra of three of these nine objects are shown in Figure 2, where the WD emission is veiled by the disc.

In the following we analyse the 31 systems in which the WD signature is well recognisable as a broad Ly $\alpha$ absorption profile. 


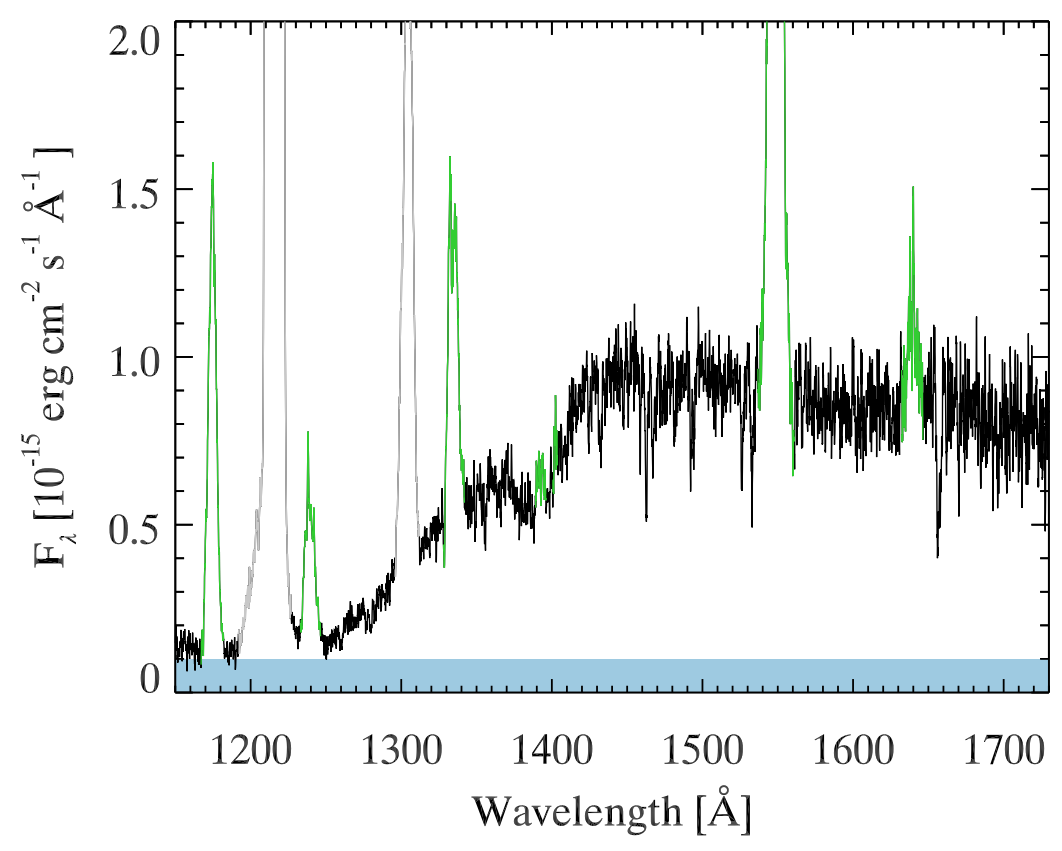

Figure 4: HST/COS spectrum of 1RXS J023238.8-371812. Plotted in green are the emission lines of C III (1175 $)$, N V (1242 $)$, C II (1335 $)$, Si IV (1400 $),$ C IV (1550 $)$ and He II (1640 $)$. The blue box highlights the presence of a second flat continuum component which, in this case, contributes $\simeq 10 \%$ of the observed flux. The geocoronal emission lines of Ly $\alpha(1216 \AA)$ and O I $(1302 \AA)$ are plotted in grey.

\section{Data analysis}

Figure 4 shows the COS data of 1RXS J023238.8-371812 as an example of a typical CV spectrum and typical signal-to-noise ratio in our sample. The blue wing of the broad Ly $\alpha$ feature extending from the short wavelength edge of the displayed spectral range to about $1450 \AA$, with the line centred at $\simeq 1216 \AA$, the quasi-molecular absorption bands of $\mathrm{H}_{2}^{+}$at $\simeq 1400 \AA$ and

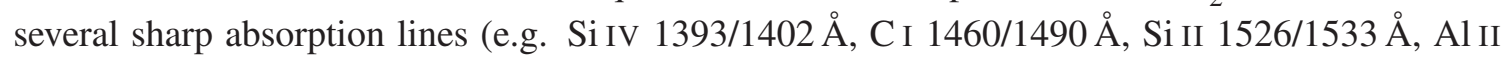
$1854 / 1862 \AA$ ) reveal the WD photosphere. The WD dominates the UV emission but the non-zero flux detected in the core of the Ly $\alpha$ suggests the presence of a secondary emission continuum component whose origin is still not clear. It has been argued that this second component could arise from the bright spot, from a hot boundary layer, or from the disc (Long et al. 1993, Godon et al. 2004, Gänsicke et al. 2005). In addition we detect emission lines, which are broadened by the Keplerian velocity distribution in the disc. A model to fit the data has to account for these three contributions (WD, second component and emission lines) observed in a CV spectrum.

We computed a grid of synthetic spectra for white dwarf atmospheres, using TLUSTY and SYNSPEC (Hubeny 1988; Hubeny \& Lanz 1995), covering $T_{\text {eff }}=10000-50000 \mathrm{~K}$ in steps of $100 \mathrm{~K}$, and metal abundances $Z$ of $0.01,0.10,0.20,0.50$ and 1.00 times their solar values. Ideally, a spectral fit to the UV data would provide both $T_{\text {eff }}$ the surface gravity $(\log g$ ). However these two quantities correlate: an increase in the temperature translates into a larger fraction of ionized hydrogen and narrower lines; this effect can be balanced by a higher gravity which increases pres- 


\begin{tabular}{|c|c|c|c|c|c|}
\hline & & $\begin{array}{c}\text { Second } \\
\text { component }\end{array}$ & $T_{\text {eff }}(\mathrm{K})$ & $d o f$ & $\chi^{2}$ \\
\hline \multirow{3}{*}{ Method 1} & Mask & blackbody & $13534 \pm 21$ & 1825 & 1768 \\
\hline & Mask & power law & $13564 \pm 21$ & 1825 & 1782 \\
\hline & Mask & constant & $13453 \pm 16$ & 1825 & 1832 \\
\hline \multirow{3}{*}{ Method 2} & Gaussian fit & blackbody & $13508 \pm 19$ & 2191 & 2195 \\
\hline & Gaussian fit & power law & $13543 \pm 19$ & 2191 & 2210 \\
\hline & Gaussian fit & constant & $13442 \pm 15$ & 2191 & 2266 \\
\hline Method 1 & & & $=57$ & & \\
\hline lethod 2 & Gaussian fit & average & $13498 \pm 51$ & & \\
\hline
\end{tabular}

Table 1: Results for 1RXS J023238.8-371812 assuming $\log g=8.35$ for the two different fitting methods for the disc emission lines: method 1, in which we mask the emission lines (Mask) and method 2, in which we include them as Gaussian profiles (Gaussian fit). The last two rows (average) report the mean and the standard deviations for each method.

\begin{tabular}{lccc}
\hline & $T_{\text {eff }}(\mathrm{K})$ & $T_{\text {eff }}(\mathrm{K})$ & $T_{\text {eff }}(\mathrm{K})$ \\
Second component & $M_{\mathrm{WD}}=0.8 \mathrm{M}_{\odot}$ & $M_{\mathrm{WD}}=0.6 \mathrm{M}_{\odot}$ & $M_{\mathrm{WD}}=0.9 \mathrm{M}_{\odot}$ \\
\hline blackbody & $13508 \pm 19$ & $12996 \pm 20$ & $13743 \pm 21$ \\
power law & $13543 \pm 19$ & $13021 \pm 16$ & $13782 \pm 21$ \\
constant & $13442 \pm 15$ & $12931 \pm 13$ & $13667 \pm 16$ \\
\hline average & $13498 \pm 51$ & $12983 \pm 46$ & $13730 \pm 59$
\end{tabular}

Table 2: $T_{\text {eff }}$ for 1RXS J023238.8-371812 allowing different $\log g$, corresponding to different WD masses. These results have been obtained fitting the data including the emission lines as Gaussian profiles, and using three different models for the second component (method 2).

sure broadening. It is not possible to break this degeneracy solely from the analysis of the COS data, therefore we needed to make an assumption on the surface gravity. Most previously published work analysing CV WD ultraviolet spectra assumed $\log g=8.00$, corresponding to $0.6 \mathrm{M}_{\odot}$, the average mass of isolated WDs (Koester et al. 1979, Liebert et al. 2005, Kepler et al. 2007), unless an independent WD mass determination was available. However, Zorotovic et al. (2011) demonstrated that the average mass of WD in CVs is actually higher than that of isolated WDs, $\simeq 0.8 \mathrm{M}_{\odot}(\log g \simeq 8.35)$. Since the canonical assumption $\log g=8.00$ does not reflect the observed average mass of CV WDs, we generated our grid of models assuming $\log g=8.35$.

To measure the effective temperature of the $31 \mathrm{CV}$ WDs, we fitted the grid of model spectra to the $H S T$ data using a $\chi^{2}$ minimisation routine. For our spectral analysis, we masked the wavelength 
regions contaminated by geocoronal emission of Ly $\alpha$ and O I $1302 \AA$. We detected the presence of an additional continuum component in all our systems, which contributes $\simeq 10-30 \%$ of the observed flux. In order to account for this additional component, we included in the fit a blackbody, a power law or a constant flux (in $F_{\lambda}$ ). To investigate the influence of the disc emission lines on our fitting procedure, we carried out our spectral analysis using two different methods: (i) method 1: we masked all the emission lines; (ii) method 2: we included the emission lines as Gaussian profiles, allowing three free parameters: line amplitude, wavelength and width.

We illustrate the differences between the two methods using 1RXS J023238.8-371812. The temperatures measured with the two methods typically only differ by $\simeq 20-30 \mathrm{~K}$ (see Table 1 ), proving that including or masking the disc lines has little influence on the fit result. Therefore, to use as much of the data as possible, we decided to include the lines in the fit using method 2.

The uncertainties of the individual fit shown in Table 1 are purely statistical, as derived from the fitting procedure, and do not reflect the real uncertainties, which are dominated by two systematic effects:

- the unknown nature of the second component: owing to the limited wavelength coverage of our data, we can not discriminate among the three second components. Including the three different additional components in the fit returns very similar temperatures for the WD. For this reason, we decided to adopt as a final $T_{\text {eff }}$ measurement, and its uncertainty, the mean and the standard deviation of the results obtained with the three different additional components. The standard deviation was always bigger than the statistical errors of the individual fits, providing a more realistic estimate for the uncertainties.

- the unknown mass: as pointed out above, temperature and mass of a WD correlate. Since the dominant source of error is the (unknown) WD mass, our assumption on $\log g$ translates into an accurate measurement of the temperature for the given mass. Releasing the assumption on the surface gravity leads to a systematic uncertainty in $T_{\text {eff }}$ of $\simeq 500 \mathrm{~K}$ (see Table 2). Henceforth, we assume $\pm 500 \mathrm{~K}$ as the final uncertainty for our $T_{\text {eff }}$ measurements. Once the masses for each system are accurately determined, it will break the degeneracy between $T_{\text {eff }}$ and $\log g$, refining our effective temperature measurements and reducing the uncertainties to the order of the standard deviation defined in the previous point.

We followed the procedure outlined above and fitted the HST data of the $31 \mathrm{CV}$ s and summarize the results in Figure 5. 


\section{Discussion}

Townsley \& Gänsicke (2009, hereafter TG09) presented a summary of CV WDs which have a reliable $T_{\text {eff }}$ determination. WD $T_{\text {eff }}$ can be measured spectroscopically, that is by fitting ultraviolet or optical data with WD atmosphere models, or photometrically, i.e. from the analysis of light curves to study the WD ingress and egress in eclipsing systems. TG09 considered a spectroscopic measurement as unreliable when the WD could not be unambiguously detected while, in the case of eclipse light curve analyses, they discarded those systems which have too low data quality or oversimplified models were used. Following these criteria, TG09 selected 43 systems with a reliable temperature measurement (see their Table 1).

Among the 43 measurements from TG09, 15 were obtained from light curve analyses (which also delivers the WD mass) or have a WD mass measurement independent from the spectral fit. For the remaining 28 objects an independent mass determination was not available and the WD $T_{\text {eff }}$ was evaluated via spectroscopic analyses, following a method similar to the one described here, assuming $\log g=8.00$. As discussed in Section 3, this assumption does not reflect the average mass in CV WDs. To combine our results with those of TG09, we need therefore to evaluate the corresponding $T_{\text {eff }}$ for $\log g=8.35$ for those 28 objects.

To do so, we fitted our HST data assuming both $\log g=8.00$ and $\log g=8.35$, and then calculated, for each object the difference in temperature $\left(\Delta T_{\text {eff }}\right)$ between $T_{\text {eff }}(\log g=8.35)$ and $T_{\text {eff }}(\log g=8.00)$. The fit to the trend of $\Delta T_{\text {eff }}$ as a function of $T_{\text {eff }}$ gives us the calibration to correct the values for those systems from TG09 which did not have an independent mass measurement, obtaining a data set consistent with our method.

Among the systems in our sample, three objects have a previous $T_{\text {eff }}$ determination in the TG09 sample: SDSS J103533.02+055158.4 (SDSS1035), CU Vel and GW Lib, which can be used for comparison with our results.

Comparing our result for SDSS1035, $T_{\text {eff }}=11630 \pm 500 \mathrm{~K}$, with the temperature measured by Littlefair et al. (2006, $\left.T_{\text {eff }}=10100 \pm 200 \mathrm{~K}\right)$, a difference of $\simeq 1500 \mathrm{~K}$ is found. This is probably due to the different methods used: Littlefair et al. derived the temperature from a colour analysis of the light curves during the WD eclipse, while we carried out a spectroscopic analysis.

A previous temperature estimation for CU Vel $\left(T_{\text {eff }}=18500 \mathrm{~K}\right)$ was obtained in 1999 by Gänsicke \& Koester from IUE spectroscopy. This temperature is higher than $T_{\text {eff }}=15342 \pm 500 \mathrm{~K}$ derived here, and it is consistent with the fact that the UV flux measured with IUE in 1999 is higher than the HST/COS flux in 2013, suggesting that the IUE observations were obtained close to an outburst.

GW Lib has been observed with $H S T$ before $\left(T_{\text {eff }}=14700 \mathrm{~K}\right.$, Szkody et al. 2002) and after $\left(T_{\text {eff }}=17300 \mathrm{~K}\right.$, Szkody et al. 2012) its superoutburst in April 2007. Since Szkody et al. (2012) assumed $\log g=8.7$, we cannot compare our $\left(T_{\text {eff }}=17051 \pm 500 \mathrm{~K}\right)$ and their result. Moreover, this is a very peculiar system which hosts a pulsating WD and, during our HST observations, it showed variations in $T_{\text {eff }}$ of $\sim 3000 \mathrm{~K}$ (see Toloza et al., this volume), therefore considerations about temperature measured at different epochs have to be done carefully.

In Figure 5 we show our sample (red circles) and the corrected effective temperatures from TG09 (triangles, blue for non-magnetic system and green for magnetic systems). While TG09 also included magnetic CVs observed in low state, we only considered non-magnetic systems. 


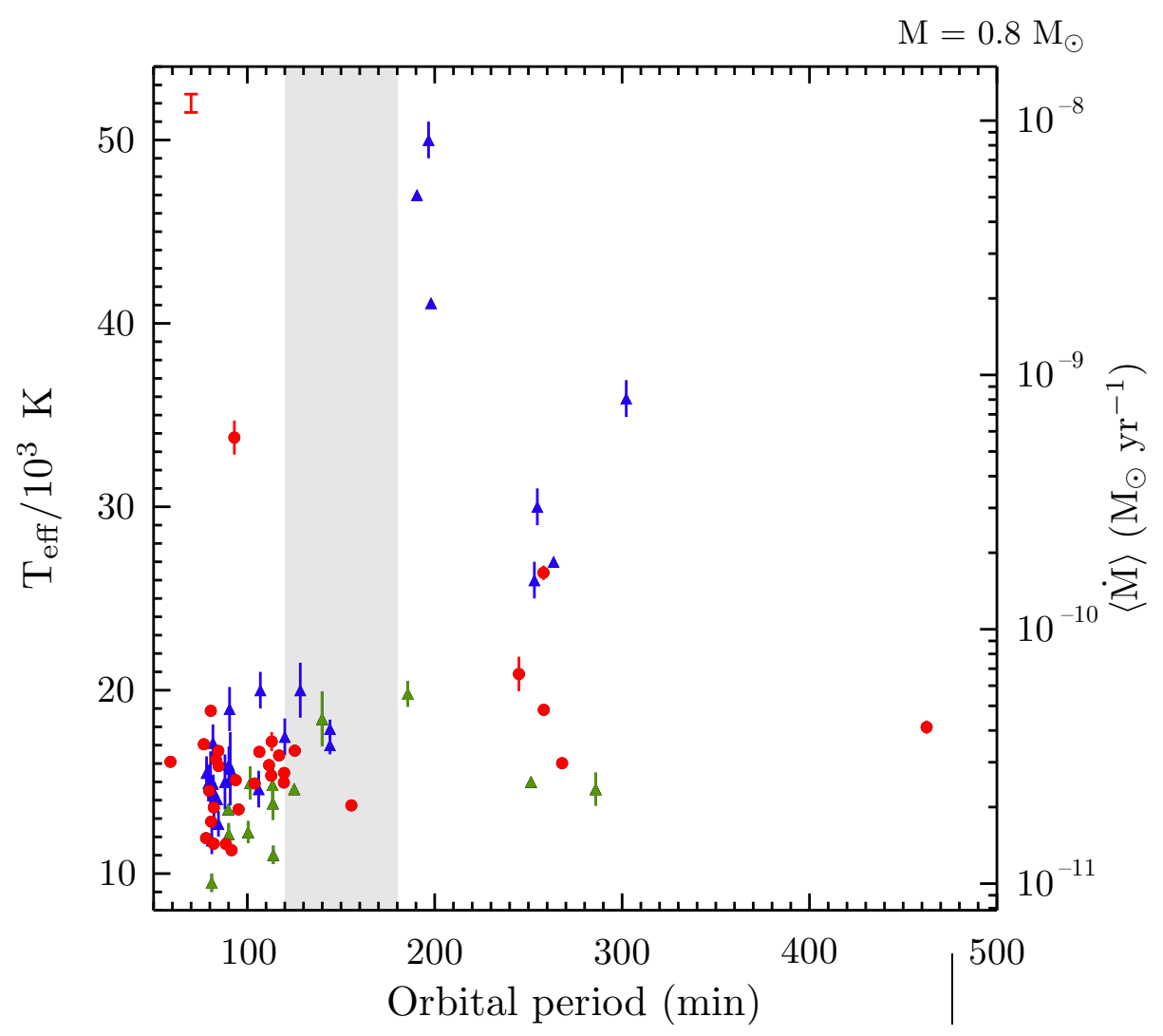

Figure 5: Effective temperature as function of the orbital period from this work (red dots) and from Townsley \& Gänsicke (2009, triangles, blue for non-magnetic systems, green for magnetic systems). The points from TG09 without errorbars are actually less accurate than the others but their uncertainty is not easily quantifiable. We only show the statistical error for our results. The systematic uncertainty in $T_{\text {eff }}$ due to the unknown mass $(\simeq 500 \mathrm{~K})$ is illustrated by the errorbar in the top left. The grey band highlights the period gap while, on the right, a mapping to $\langle\dot{M}\rangle$ calculated through equation 1 from TG09 is shown for $M=0.8 \mathrm{M}_{\odot}$.

The uncertainties in the two samples are dominated by the unknown WD mass. It is important to notice that these uncertainties are on the order of $\sim 500-1000 \mathrm{~K}$ (see Section 3), therefore even when the WD masses will be accurately determined, the shape of the distribution will not drastically change.

Since $T_{\text {eff }}$ is set by accretion heating, the distribution in Figure 5 can be compared to models for the present day CV population in the $\dot{M}-P_{\text {orb }}$ plane (see Figure $2 \mathrm{~b}$ from Goliasch \& Nelson 2015). Our results agree well overall with the theoretical predictions, with the evolution of long period systems driven by magnetic braking with a typical mass transfer rate of $\dot{M} \sim 10^{-10}$ to $\sim 10^{-9} \mathrm{M}_{\odot} \mathrm{yr}^{-1}$. In contrast, systems below the period gap driven only by gravitational wave radiation are characterised by a lower average $\dot{M} \sim 5 \times 10^{-11} \mathrm{M}_{\odot} \mathrm{yr}^{-1}$.

At $\mathrm{P}_{\text {orb }} \sim 180-240 \mathrm{~min}$, several systems with $T_{\text {eff }} \simeq 40000-50000 \mathrm{~K}$ are present. These objects are known as VY Scl systems and are characterised by high temperatures, which indicate high accretion rates onto the WD, with $\dot{M}$ about ten times above than the value predicted by the 
standard evolution theory (TG09, Howell et al. 2001). Goliasch \& Nelson (2015) argued that these objects could arise naturally from systems close to the regime of unstable mass transfer - where the mass of the donor star is similar to the WD mass - but this hypothesis needs to be investigated in more detail.

Below the period gap, the measured effective temperatures show the expected trend, with a monotonic decrease towards the period minimum. However, they also show some unexpected features. First, they present a larger scatter than predicted by theory, where the majority of the systems are clustered at the period minimum whereas our data present a larger temperature spread near $P_{\text {orb }} \simeq 80 \mathrm{~min}$. Second, the population synthesis results show the presence of two narrow tracks according to the WD core composition (He or CO) while, at present, no CV with a He-core WD has been identified. The last discrepancy could arise from an incompleteness in the theoretical binary population synthesis models. As suggested by Schreiber et al. (2016), the problem of the absence of low-mass CV WDs could be solved taking into account a consequential angular momentum loss, which would be more efficient for lower WD masses. This model could explain why no He-core WD have been discovered, resolving another disagreement between observations and theory.

Finally, reaching the minimum period, CVs are expected to evolve back towards longer periods with mean accretion rates on the order of $\dot{M} \sim 10^{-11} \mathrm{M}_{\odot} \mathrm{yr}^{-1}$, corresponding to $T_{\text {eff }} \lesssim 10000 \mathrm{~K}$. These are the so-called "period bouncers" and the standard model of CV evolution predicts that about $70 \%$ of the present CVs should be observed in this phase. Our results show an absence of these systems, suggesting that none of them are strong period bouncer candidates and that a major component of the predicted $\mathrm{CV}$ population still remains elusive to observations.

\section{Conclusions}

We presented the results from a large $H S T / \mathrm{COS}$ survey of 40 cataclysmic variables. We selected 31 systems in which the WD signature dominates the UV flux as revealed by a broad Ly $\alpha$ profile, and we fitted the $H S T$ data with atmosphere models to measure the WD effective temperatures. Combining our results to the $43 \mathrm{CV}$ s from TG09, we almost doubled the number of objects with an accurate temperature measurement. Comparing the $T_{\text {eff }}-P_{\text {orb }}$ distribution with the mean accretion rate $\dot{M}-P_{\text {orb }}$ distribution from population models, we find a general agreement with the theoretical predictions, but some clear differences are evident which could arise from observational biases and/or an incomplete understanding of $\mathrm{CV}$ formation and evolution.

\section{Acknowledgments}

The research leading to these results has received funding from the European Research Council under the European Union's Seventh Framework Programme (FP/2007-2013) / ERC Grant Agreement n. 320964 (WDTracer).

Support for program 12870 was provided by NASA through a grant from the Space Telescope Science Institute, which is operated by the Association of Universities for Research in Astronomy, Inc., under NASA contract NAS 5-26555. 
We gratefully acknowledge the variable star observations from the AAVSO International Database contributed by observers worldwide and used in this research. We are indebted to the global amateur community for their outstanding support, which made the HST survey possible.

\section{References}

Epelstain N., Yaron O., Kovetz A., Prialnik D., 2007, MNRAS, 374, 1449

Gänsicke B. T., Beuermann K., 1996, A\&A, 309, L47

Gänsicke B. T., Koester D., 1999, A\&A, 346, 151

Gänsicke B. T., Long K. S., Barstow M. A., Hubeny I., 2006, ApJ, 639, 1039

Gänsicke B. T., Szkody P., Howell S. B., Sion E. M., 2005, ApJ, 629, 451

Godon P., Sion E. M., Cheng F. H., Szkody P., Long K. S., Froning C. S., 2004, ApJ, 612, 429

Goliasch J., Nelson L., 2015, ApJ, 809, 80

Howell S. B., Nelson L. A., Rappaport S., 2001, The Astrophysical Journal, 550, 897

Hubeny I., 1988, Computer Physics Communications, 52, 103

Hubeny I., Lanz T., 1995, ApJ, 439, 875

Kepler S. O., Kleinman S. J., Nitta A., Koester D., Castanheira B. G., Giovannini O., Costa A. F. M., Althaus L., 2007, MNRAS, 375, 1315

King A. R., 1997, MNRAS, 288, L16

Koester D., Schulz H., Weidemann V., 1979, A\&A, 76, 262

Koester D., Weidemann V., Zeidler-K.T. E. M., Vauclair G., 1985, A\&A, 142, L5

Liebert J., Bergeron P., Holberg J. B., 2005, ApJS, 156, 47

Littlefair S. P., Dhillon V. S., Marsh T. R., Gänsicke B. T., Southworth J., Baraffe I., Watson C. A., Copperwheat C., 2008, MNRAS, 388, 1582

Littlefair S. P., Dhillon V. S., Marsh T. R., Gänsicke B. T., Southworth J., Watson C. A., 2006, Science, 314,1578

Long K. S., Blair W. P., Bowers C. W., Davidsen A. F., Kriss G. A., Sion E. M., Hubeny I., 1993, ApJ, 405, 327

Nelan E. P., Wegner G., 1985, ApJ, 289, L31

Osaki Y., 1974, PASJ, 26, 429

Paczynski B., Sienkiewicz R., 1983, The Astrophysical Journal, 268, 825 
Patterson J., 1984, ApJS, 54, 443

Piro A. L., Arras P., Bildsten L., 2005, ApJ, 628, 401

Prialnik D., Kovetz A., 1995, ApJ, 445, 789

Rappaport S., Verbunt F., Joss P. C., 1983, ApJ, 275, 713

Ritter H., 1987, Mem. Soc. Astron. Italiana, 58, 133

Ritter H., Kolb U., 2003, A\&A, 404, 301

Schreiber M. R., Zorotovic M., Wijnen T. P. G., 2016, MNRAS, 455, L16

Sion E. M., Gänsicke B. T., Long K. S., Szkody P., Knigge C., Hubeny I., de Martino D., Godon P., 2008, ApJ, 681, 543

Slevinsky R. J., Stys D., West S., Sion E. M., Cheng F. H., 1999, PASP, 111, 1292

Spruit H. C., Ritter H., 1983, A\&A, 124, 267

Szkody P., Gänsicke B. T., Howell S. B., Sion E. M., 2002, ApJ, 575, L79

Szkody P., Mukadam A. S., Gänsicke B. T., Henden A., Sion E. M., Townsley D., Chote P., Harmer D., Harpe E. J., Hermes J. J., Sullivan D. J., Winget D. E., 2012, ApJ, 753, 158

Szkody P., Sion E. M., Gänsicke B. T., Howell S. B., 2002, in Gänsicke B. T., Beuermann K., Reinsch K., eds, The Physics of Cataclysmic Variables and Related Objects Vol. 261 of Astronomical Society of the Pacific Conference Series, The temperatures of white dwarfs in CVs: Implications of results from HST. p. 21

Townsley D. M., Bildsten L., 2003, The Astrophysical Journal, 596, L227

Townsley D. M., Gänsicke B. T., 2009, The Astrophysical Journal, 693, 1007

Warner B., 1973, MNRAS, 162, 189

Warner B., 1995, Cambridge Astrophysics Series, 28

Zorotovic M., Schreiber M. R., Gänsicke B. T., 2011, A\&A, 536, A42 